\title{
A Uniqueness Result for a Simple Superlinear Eigenvalue Problem
}

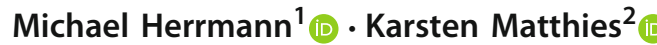

Received: 6 June 2020 / Accepted: 3 February 2021 / Published online: 23 February 2021

(c) The Author(s) 2021

\begin{abstract}
We study the eigenvalue problem for a superlinear convolution operator in the special case of bilinear constitutive laws and establish the existence and uniqueness of a oneparameter family of nonlinear eigenfunctions under a topological shape constraint. Our proof uses a nonlinear change of scalar parameters and applies Krein-Rutman arguments to a linear substitute problem. We also present numerical simulations and discuss the asymptotics of two limiting cases.
\end{abstract}

Keywords Nonlinear eigenvalue problems · Nonlocal coherent structures ·

Krein-Rutman theorems · Asymptotic analysis of nonlinear integral operators

Mathematics Subject Classification 45G10 · 45M05 - 47J10

\section{Introduction}

Nonlinear analogues to linear eigenvalue problems arise in many branches of the sciences and often model coherent structures in spatially extended dynamical systems. Examples will be discussed below in greater detail and include traveling waves in Hamiltonian lattices as well as certain nonlocal aggregation models. Nonlinear eigenvalue equations often combine nonlinear superpositions with linear pseudo-differential

Communicated by Arnd Scheel.

$\bowtie$ Michael Herrmann

michael.herrmann@tu-braunschweig.de

$\bowtie \quad$ Karsten Matthies

k.matthies@bath.ac.uk

1 Institut für Partielle Differentialgleichungen, Technische Universität Braunschweig,

Universitätzplatz 2, 38106 Brunswick, Germany

2 Department of Mathematical Sciences, University of Bath, Bath BA2 7AY, UK 
operators, where the latter can represent convolutions, spatial derivatives, or the solution of an elliptic auxiliary problem.

A simple and spatially one-dimensional prototype is the scalar equation

$$
\sigma u=a * f(u)
$$

which involves a localized convolution kernel $a$ and a given nonlinear function $f$. The problem is to find solution pairs $(\sigma, u)$ consisting of a real eigenvalue $\sigma$ and a nontrivial scalar eigenfunction $u$ which might be of periodic or homoclinic type. As explained below, the existence of solutions to (1) can be established by many different approaches and this has already been done for special choices or certain classes of $a$ and $f$. The uniqueness problem, however, is notoriously difficult in the superlinear case, and we are not aware of any rigorous result that applies to (1).

As a first step toward a more general uniqueness theory, we study in this paper the case of bilinear functions $f$ which are piecewise linear with two slopes. This simplifying assumption allows us to transform (1) into the linear eigenvalue problem for a modified convolution operator with cut-off parameter $\xi$. We further suppose that both the kernel $a$ and the eigenfunction $u$ are nonnegative, even, and unimodal. This shape constraint provides an existence and uniqueness result for the linear substitute problem thanks to a variant of the classical Krein-Rutman theorem. Moreover, it ensures that the nonlinear relation between $\sigma$ and $\xi$ is bijective. The combination of both arguments implies a uniqueness result as well as a novel existence proof for the nonlinear eigenvalue problem (1).

Before we specify our assumptions and findings, we continue with an informal overview on possible applications and give a more detailed discussion of the underlying mathematical problems.

\section{Hamiltonian Lattice Waves}

Traveling waves in Hamiltonian lattices can be viewed as the nonlinear analogues to plane-wave excitations and provide the building blocks for more complex solution patterns. The prototypical example is Fermi-Pasta-Ulam-Tsingou (FPUT) chains with nearest neighbor interactions. There, spatially discrete waves propagate and satisfy the advance-delay differential equation

$$
\sigma u^{\prime \prime}(x)=f(u(x+1))+f(u(x-1))-2 f(u(x))
$$

see for instance (Friesecke and Wattis 1994; Filip and Venakides 1999; Herrmann 2010) for more background information. Here, $\sigma$ is the squared wave speed and $u$ denotes the unknown profile function for the atomic distances. Moreover, $x$ stands for the spatial variable in the co-moving frame and $f$ abbreviates the nonlinear stressstrain relation, which is usually given by the derivative of an interaction potential. After twofold integration with respect to $x$-and eliminating the constants of integration by assuming a homoclinic wave profile-Eq. (2) can be transformed into the eigenvalue 
problem (1) with a tent-map kernel, see Eq. (10) below. Another, but closely related, example is the first-order equation

$$
\sigma u^{\prime}(x)=f(u(x+1))-f(u(x-1))
$$

which describes the traveling waves in a Hamiltonian semidiscretization of scalar conservation laws, see (Herrmann 2012). It is likewise equivalent to (1), but now equipped with the piecewise constant kernel (11).

For FPUT chains, the existence of periodic or solitary waves has been established in different frameworks and the employed methods include critical point techniques (Pankov 2005), constrained optimization (Friesecke and Wattis 1994; Filip and Venakides 1999; Herrmann 2011; Herrmann and Matthies 2020), and asymptotic or perturbative arguments (Friesecke and Pego 1999; Iooss and James 2005; James 2012). More recent work in this field concerns waves in lattice systems with a finite or even infinite number of interaction bonds and vector-valued displacement fields. The corresponding advance-delay differential equations can also be written in the form of nonlinear eigenvalue problems but include more than one convolution kernel and several nonlinearities, see Herrmann and Mikikits-Leitner (2016), Chen and Herrmann (2018), Pego and Van (2019) and Herrmann and Matthies (2019a).

Although the existence theory of Hamiltonian lattice waves developed quite well, very little is known about their uniqueness, parameter dependence, and dynamical stability. The only rigorous results either concern completely integrable cases (Toda chain) or are restricted to asymptotic regimes, in which the problem can be tackled as a singular perturbation of an underlying ODE regime, see Friesecke and Pego (1999) and Herrmann and Matthies (2019b) for the limits of small and large wave speeds, respectively. Any progress in this direction would enhance our understanding of energy transport in quasilinear Hamiltonian systems with strong dispersion. Of particular interest are the lattice variants of Whitham's modulation theory and their predictions on the creation and propagation of dispersive shock waves in spatially discrete media. For instance, for FPUT chains with superquadratic force function $f$ we expect the existence of a four-parameter family of periodic and linearly stable lattice waves, which can be modulated according to a hyperbolic system of four nonlinear conservation laws, see for instance (Filip and Venakides 1999; Dreyer et al. 2006; Dreyer and Herrmann 2008; Yasuda et al. 2017). There exists strong numerical evidence for the existence of modulated traveling waves, but we still lack a rigorous understanding for nonintegrable cases. An important subproblem in this context is to characterize the solution set of (1) for at least special kernels and the spectral properties of its linearization.

We further emphasize that standing waves or breather solutions in lattice system of coupled oscillators can also be linked to nonlinear eigenvalue problems although the corresponding equations are often semilinear and involve discrete convolution operators. A typical example is

$$
\sigma u_{j}=\alpha\left(u_{j+1}+u_{j-1}\right)+f\left(u_{j}\right)
$$

and appears in the theory of discrete nonlinear Schrödinger equations. Solutions can be constructed by variational techniques or asymptotic methods, see for instance 
(MacKay and Aubry 1994; Weinstein 1999; Herrmann 2011) and the overview in Kevrekidis (2009), but it seems that there is no global theory concerning the uniqueness of solutions for the relevant classes of nonlinearities (which might be either focusing or defocusing).

\section{Further Related Applications}

Many aggregation models in mathematical biology also involve both convolution operators and nonlinearities. A first example is the nonlocal parabolic PDE

$$
\partial_{t} \varrho=\Delta_{x}(h(a * \varrho) \varrho)
$$

which has been introduced and studied in Burger et al. (2013), Haškovec and Oelz (2015) to model the pattern forming effect of concentration dependent diffusion mobilities. The scalar function $h$, which is supposed to be nonnegative and decreasing, describes that the tendency of biological individuals to undertake a random walk depends on the local population density $\varrho(t, x)$, where the convolution kernel reflects the corresponding interaction domain. Despite its apparent simplicity, the equation exhibits a rather intriguing dynamics even in one space dimension. Starting with random initial data one observes the rather rapid formation of metastable peaks, which in turn interact and annihilate each other in a coarsening dynamics on much larger time scales. Each localized peak can be viewed as a stationary state and the corresponding equation $h(a * \varrho) \varrho=c$ is equivalent to the eigenvalue problem (1) via the substitution $u=a * \varrho, f(u)=1 / h(u)$, and $\sigma=1 / c$. It is desirable to understand the complete set of metastable states and their spectral properties with respect to the linearized parabolic dynamics.

A second example in one space dimension is the Wasserstein gradient flow

$$
\partial_{t} \varrho=\partial_{x}\left(\varrho \partial_{x}(g(\varrho)-a * \varrho)\right)
$$

where $g$ is now a strictly increasing function, see (Burger et al. 2014; Kaib 2017) and references therein. The nonlinear eigenvalue problem (1) yields again stationary states with $u=g(\varrho)+c=a * \varrho$ provided that $\sigma^{-1} f$ is the inverse function to $g+c$. However, there exist more general steady states $\varrho$ which are compactly supported and satisfy the nonlinear integral equation on that support only, see Burger et al. (2014). For linear functions $g$, the existence and uniqueness of stationary solutions with compact support have been studied in Burger et al. (2013). The corresponding equation is also naturally related to our linear substitute problem and will be discussed at the end of Sect. 2.

The scalar and one-dimensional equation (1) can also be viewed as a simplified toy model for other nonlinear eigenvalue problems. For instance, chimera states in the Kuramoto equation can be characterized by a complex-valued analogue to (1) as explained in Omel'chenko et al. (2008), Omel'chenko (2018). Further examples are the nonlocal variants of the Allan-Cahn equation or systems of reaction diffusion equations. In this context, however, the nonlinearities are typically bistable instead 
of monotone and the equation for the relevant coherent states often involves additional derivative terms that account for a possible propagation with constant speed, see for instance (Faye and Scheel 2015; Bakker and Scheel 2018). The existence and uniqueness problem is hence more challenging and has already been investigated by perturbative arguments as described in Anderson et al. (2016), Scheel and Tao (2019). Finally, many semilinear elliptic PDE can be interpreted as nonlinear eigenvalue problems, where $a$ is the order preserving solution operator of the underlying linear problem. The standard example in one space dimension is the exponential kernel $a(x)=\frac{1}{2} \exp (-|x|)$, which can be viewed as the fundamental solution to the differential operator $-\partial_{x}^{2}+1$. The corresponding eigenvalue problem (1) can be tackled by planar ODE techniques applied to $-\sigma u^{\prime \prime}=f(u)-\sigma u$ and this provides a complete characterization of all solutions. For general kernels $a$, however, we lack such a simplifying reinterpretation. Notice also that the space variable $x$ in elliptic PDEs is often confined to a certain subdomain of $\mathbb{R}^{n}$, which requires to impose additional boundary conditions for $u$.

\section{Problems, Discussion, and Outlook}

A complete mathematical theory of nonlinear eigenvalue problems should address the existence, uniqueness, and parameter dependence of solutions but also the spectral properties of its linearization as those naturally appear in the corresponding continuation and bifurcation analysis. All available rigorous and heuristic results indicate that the mathematical theory strongly depends on the structural properties of the convolution kernel and the nonlinearities.

In this paper we restrict our considerations to one space dimension, unimodal kernels $a$, and a piecewise linear function $f$ being superlinear. This setting is relevant for both Hamiltonian lattice waves and biological aggregation models and still related to many unsolved mathematical problems concerning the uniqueness and dynamical stability of solutions. The case of sublinear functions $f$ should be simpler because both existence and uniqueness results can be inferred from the abstract theory in Rabinowitz (1971). The case of nonmonotone functions $f$ is of course much more involved and will not be addressed here. Furthermore, we do not discuss general convolution kernels (being nonnegative or not) or the case of vector-valued eigenfunctions.

The existence of periodic or solitary solutions to (1) can be established by various methods and we already mentioned variational and asymptotic techniques for special classes of $a$ and $f$. For sufficiently nice kernels $a$, the existence of periodic solutions can also be deduced from the classical Crandall-Rabinowitz theory (Crandall and Rabinowitz 1971), which starts with a periodic solution to the linear equation

$$
\sigma_{0} u_{0}=f^{\prime}(0) a * u_{0}
$$

and provides a global bifurcation result under natural nondegenericity conditions. In one space dimension, all existence conditions can easily be checked by means of Fourier arguments. We are, however, not aware of any related uniqueness result that covers superlinear functions $f$ and is not based on local bifurcation analysis. 
Motivated by numerical simulations and the existing results for special cases we conjecture - both for any fixed periodicity length $L<\infty$ and the solitary limit $L=$ $\infty$-the existence of a unique family of solutions $(\sigma, u)$ that can be parameterized by the eigenvalue $\sigma$ and involves eigenfunctions $u$ that are nonnegative, even, and unimodal (see below for a precise definition). This statement does not exclude the existence of further eigenfunctions that do not meet the shape constraint and can be interpreted as a nonlinear version of the famous Krein-Rutman theorem (or its Perron-Frobenius analogue for matrices). There already exist some nonlinear variants of these theorems, see for instance (Mahadevan 2007; Arapostathis 2018), but these are restricted to 1-homogeneous nonlinearities which are necessarily linear in the scalar case considered here.

Moreover, except for the exponential kernel it is not clear how to characterize all solutions $(\sigma, u)$ to the linearized eigenvalue problem

$$
\sigma_{*} u+\sigma u_{*}=a *\left(f^{\prime}\left(u_{*}\right) u\right)
$$

where $\left(\sigma_{*}, u_{*}\right)$ denotes a fixed solution to the nonlinear equation. Preliminary numerical simulations indicate that there should be some analogue to the Sturm-Liouville theory for second-order ODEs, but it seems that there is no rigorous result in this direction that applies to arbitrary kernels $a$.

With the present paper, we wish to contribute to the general theory of the eigenvalue problem (1) with superlinear $f$. At the moment we are only able to prove our conjecture for piecewise linear functions $f$ but hope to investigate more general nonlinearities in a forthcoming study. The next natural step is to allow $f^{\prime}$ to exhibit more than one jump discontinuity, but even the trilinear case is, as far as we see, considerably more involved than the bilinear situation studied here. We also postpone the investigation of the linearized eigenvalue problem (5) or related equations to future work.

\section{Assumptions and Results}

As illustrated in Fig. 1, we consider functions $f$ that are piecewise linear on the interval $[0, \infty)$. More precisely, we set

$$
f(r):= \begin{cases}\zeta r & \text { for } \quad r \in[0, \theta], \\ (\zeta+\eta)(r-\theta)+\zeta \theta & \text { for } r \in[\theta, \infty)\end{cases}
$$

and assume that the three free parameters $\zeta, \theta, \eta$ comply with the superlinearity condition

$$
\zeta \geq 0, \quad \theta>0, \quad \eta>0 .
$$



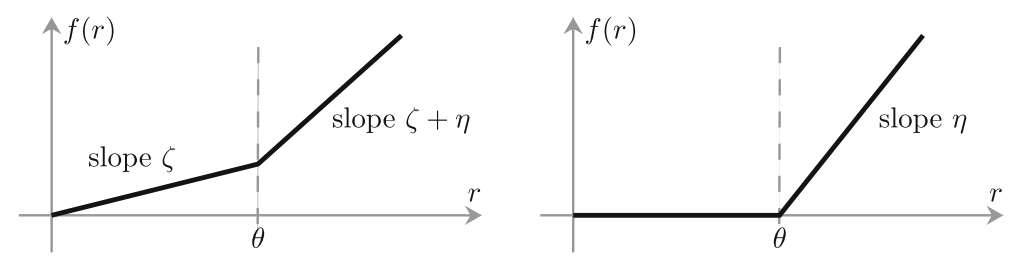

Fig. 1 The bilinear function $f$ from (6) as studied in this paper in the superlinear parameter regime (7). The general case (left panel) can be traced back to the special case $\zeta=0$ (right panel) according to Proposition 3

It is also essential for our uniqueness result that the kernel $a: \mathbb{R} \rightarrow \mathbb{R}$ is integrable and belongs to the cone

$$
\begin{gathered}
\mathcal{U}=\left\{u \in \mathrm{L}^{2}(\mathbb{R}): u(x)=u(-x) \text { and } u(x) \geq u(\tilde{x}) \geq 0\right. \\
\text { for almost all } 0<x<\tilde{x}\},
\end{gathered}
$$

which contains all bounded $\mathrm{L}^{2}$-functions that are even, nonnegative, and unimodal. We suppose further that $a$ is differentiable and strictly unimodal according to

$$
a^{\prime}(x)<0 \text { for } x>0
$$

because this simplifies the presentation. However, we always discuss how more general results can be obtained by relaxing the precise formulation of theorems or enhancing the technical arguments in their proofs.

Assumption 1 (Properties of the convolution kernel) The convolution kernel $a$ belongs to $\mathcal{U}$ and $L^{1}(\mathbb{R})$ is normalized by

$$
\int_{-\infty}^{+\infty} a(x) \mathrm{d} x=1,
$$

and satisfies additionally (8).

A prototypical example for Assumption 1 is the Gaussian

$$
a_{1}(x):=\exp \left(-x^{2}\right) / \sqrt{\pi}
$$

while the unimodal tent map

$$
a_{2}(x):=\max \{0,1-|x|\}
$$

and the indicator function

$$
a_{3}(x):= \begin{cases}1 & \text { for }|x| \leq \frac{1}{2} \\ 0 & \text { else }\end{cases}
$$



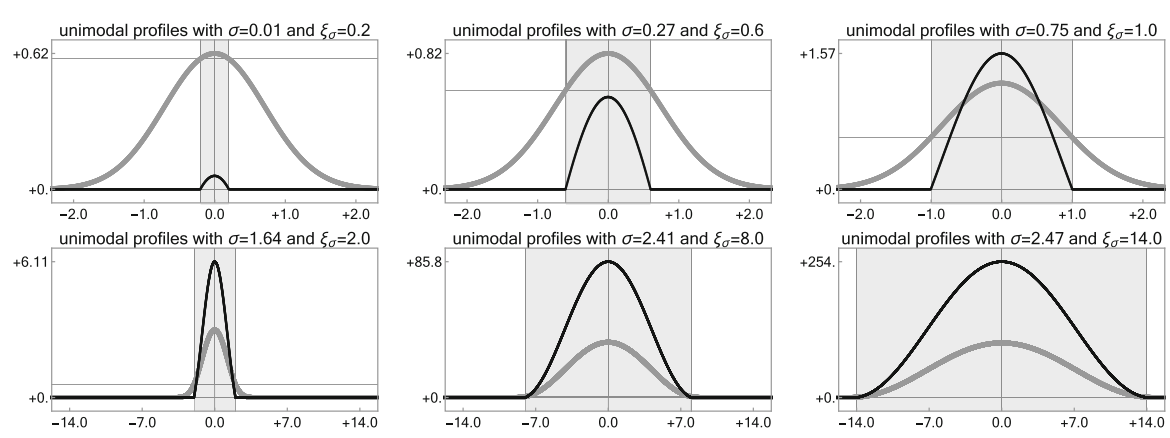

Fig. 2 Numerical profiles $u_{\sigma}$ (gray) and $f\left(u_{\sigma}\right)$ (black) for several values of $\sigma$, the regular kernel (9), and nonlinearity parameters $\zeta=0, \theta=0.6, \eta=2.5$. The gray box represents the interval $I_{\xi_{\sigma}}$ which is defined by $u_{\sigma}\left( \pm \xi_{\sigma}\right)=\theta$. Notice the different plot regions for top and bottom row
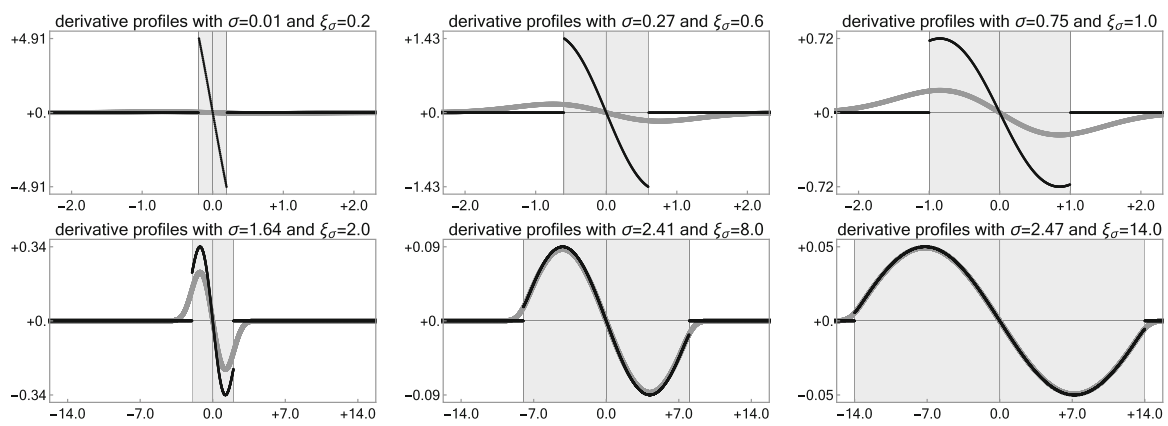

Fig. 3 Effective derivative profile $v_{\sigma}=\chi_{\xi_{\sigma}} \cdot u_{\sigma}^{\prime}$ (gray) as well as $a * v_{\sigma}=\eta^{-1} \sigma u_{\sigma}^{\prime}$ (black) for the nonlinear eigenfunctions from Fig. 2. The Krein-Rutman eigenfunction provided by Proposition 1 for $\xi_{\sigma}$ is proportional to $v_{\sigma}$

are compactly supported and violate (8). Both kernels are nonetheless interesting since they are naturally related to traveling waves in FPUT chains and other spatially discrete conservation laws, see (2) and (3). For the kernel (10), the eigenvalue problem (1) with bilinear function (6) has already been solved in Truskinovsky and Vainchtein (2014) by a combination of Fourier and numerical methods, see the more detailed comments at the end of Sect. 3.

Our findings for the nonlinearity (6) and kernels as in Assumption 1 can be summarized as follows.

Main Result 1 (Existence and uniqueness under shape constraint for bilinear nonlinearities)

1. There exists a unique one-parameter family of nonlinear eigenfunction $u_{\sigma} \in \mathcal{U}$ parameterized by $\zeta<\sigma<\zeta+\eta$.

2. Formal asymptotic expansions suggest the following limit behavior: 

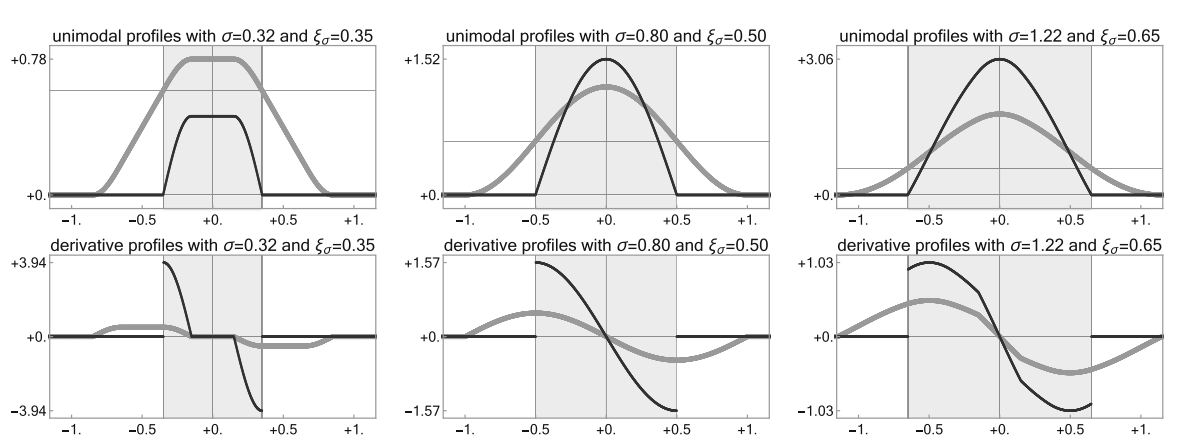

Fig. 4 Numerical simulations for the degenerate kernel (11) and nonlinearity parameters as in Fig. 2. The statement of some results must be modified in this case since the strict unimodality condition (8) is not satisfied

(a) The eigenfunctions $u_{\sigma}$ converge as $\sigma \searrow \zeta$ to a nontrivial limit profile $u_{\zeta} \in \mathcal{U}$ with

$$
u_{\zeta}(x) \leq \theta \text { for all } x, \quad u_{\zeta}(0)=\theta
$$

but the details are different for $\zeta=0$ and $\zeta>0$.

(b) There is no limit $u_{\zeta+\eta}$ due to $\lim _{\sigma \nearrow \zeta+\eta}\left\|u_{\sigma}\right\|_{2}=\infty$.

The rigorous part of our main result is proven in Proposition 4, while the nonrigorous asymptotic analysis is presented in the appendix. We also refer to Figs. 2, 3, and 4 for numerical simulations with $\zeta=0$ and emphasize that our results do not exclude the existence of further eigenfunctions outside the cone $\mathcal{U}$. In addition to the trivial nonuniqueness due to the shift invariance of (1), we expect the existence of families of periodic solutions. Moreover, for rapidly decaying kernels one might think about multi-bump solutions, which can be imagined as superposition of finitely many and well-separated single-bump solutions having unimodal profile.

\section{Proof Strategy and Organization of Paper}

The central ideas for the proof of our main results can be sketched as follows. Assuming smoothness of $u$ we deduce $u^{\prime} \in \mathcal{N}$, where

$$
\mathcal{N}:=\left\{v \in \mathrm{L}^{2}(\mathbb{R}): v(x)=-v(-x) \text { and } v(x) \leq 0 \text { for almost all } x>0\right\}
$$

contains all odd functions that attain nonpositive values on $(0, \infty)$ (usually, elements of $-\mathcal{N}$ and $\mathcal{N}$ are called positive and negative, respectively). Moreover, differentiating (1) we find

$$
\sigma u^{\prime}=a *\left(\zeta u^{\prime}+\eta \chi \xi \cdot u^{\prime}\right)
$$


with

$$
I_{\xi}:=[-\xi, \xi], \quad \chi \xi(x):= \begin{cases}1 & \text { for } x \in I_{\xi} \\ 0 & \text { else }\end{cases}
$$

where $\xi$ is the value at which $u$ attains the value $\theta$. This reads

$$
\theta=u(\xi)=-\int_{\xi}^{\infty} u^{\prime}(x) \mathrm{d} x
$$

In the special case of $\zeta=0$, the necessary condition (12) reads

$$
\lambda u^{\prime}=a * v
$$

and involves the effective derivative profile

$$
v=\chi \xi \cdot u^{\prime}
$$

as well as

$$
\lambda=\sigma / \eta
$$

Formula (14) implies the linear eigenvalue problem

$$
\lambda v=\mathcal{A}_{\xi} v
$$

where

$$
\mathcal{A}_{\xi} v:=\chi_{\xi} \cdot\left(a *\left(\chi_{\xi} \cdot v\right)\right)
$$

is a compact and symmetric operator, which exhibits nice invariance properties. In particular, adapting the strong version of the Krein-Rutman theorem we can show that the eigenspace to the largest eigenvalue of $\mathcal{A}_{\xi}$ is simple and generated by an element of $\mathcal{N}$, see Proposition 1 . This observation yields in combination with (13) the existence and uniqueness of a family of solutions to (1) which is naturally parameterized by $\xi$ and can be computed efficiently. Moreover, a closer inspection of the linear eigenvalue problem (17) as carried out in Proposition 2 reveals that the mapping $\xi \mapsto \lambda \mapsto \sigma$ is invertible so that $\xi$ can be replaced by $\sigma$. This change of parameters is the nonlinear part of our approach.

Another observation extends the existence and uniqueness result to the general case $\zeta>0$. More precisely, we identify in Proposition 3 a modified kernel $\tilde{a} \in \mathcal{U}$ with $\int_{\mathbb{R}} \tilde{a}(x) \mathrm{d} x=1$ such that the implication

$$
w-\mu a * w=a * g \Longleftrightarrow w=(1-\mu)^{-1} \tilde{a} * g
$$


holds with $\mu=\sigma^{-1} \zeta \in(0,1)$. This enables us to transform the general case $\zeta>0$ into the special case $\zeta=0$ by replacing the kernel $a$ with $\tilde{a}$ and changing $\sigma$. A similar argument applies on the level of the derivatives because (12) can be written as $\tilde{\lambda} u^{\prime}=\tilde{a} *\left(\chi_{\xi} \cdot u^{\prime}\right)$.

\section{Linear Eigenvalue Problem for the Effective Derivative Profile}

In this section we study the eigenvalue problem of the operator $\mathcal{A}_{\xi}$ from (18) in the space $L_{\text {odd }}^{2}(\mathbb{R})$ which contains all functions that are square-integrable and odd. Notice that the representation formulas

$$
\begin{aligned}
\left(\mathcal{A}_{\xi} v\right)(x) & =\int_{-\xi}^{+\xi} a(x-y) v(y) \mathrm{d} y \\
& =\int_{0}^{\xi}(a(x-y)-a(x+y)) v(y) \mathrm{d} y \text { for }|x| \leq \xi
\end{aligned}
$$

and

$$
\left(\mathcal{A}_{\xi} v\right)(x)=0 \text { for }|x|>\xi
$$

hold for any $v \in \mathrm{L}_{\text {odd }}^{2}(\mathbb{R})$.

Lemma 1 (Properties of the modified convolution operator) For any $\xi \in(0, \infty)$, the operator $\mathcal{A}_{\xi}$, maps $L^{2}(\mathbb{R})$ into itself, respects the even-odd parity, and is both compact and self-adjoint. Moreover, the convex cones $\mathcal{N}$ and $\mathcal{U}$ are invariant under the action of $\mathcal{A}_{\xi}$.

Proof The first assertions can be derived from (18) using standard arguments for convolution and multiplication operators. Moreover, since $a$ is even and unimodal, the invariance of $\mathcal{N}$ and $\mathcal{U}$ follows from (20) and the analogous formula in $L_{\text {even }}^{2}(\mathbb{R})$.

\section{Linear Uniqueness Result}

As first main auxiliary result we prove that the largest eigenvalue of $\mathcal{A}_{\xi}$ is simple and spanned by a unique normalized eigenfunction in $\mathcal{N}$. Such uniqueness results are usually inferred from the strong version of the Krein-Rutman theorem, but the classical formulation requires an order preserving operator that maps the cone of positive (or negative) elements in its interiors, see for instance [Dautray and Lions (1990), appendix to chapter IIX]. In our case, however, the cone $\mathcal{N}$ has no inner points and the compact support of $\chi_{\xi}$ implies for every $v \in \mathcal{N}$ that $\mathcal{A}_{\xi} v$ belongs to the topological boundary of $\mathcal{N}$ in any reasonable topology. 
The assertions of the Krein-Rutman Theorem hold notwithstanding. The crucial idea is that any eigenfunction that corresponds to the largest eigenvalue of $\mathcal{A}_{\xi}$ must belong to the smaller cone

$$
\begin{gathered}
\tilde{\mathcal{N}}_{\xi}:=\left\{v \in \mathcal{N} \cap C^{1}\left(I_{\xi}\right): v^{\prime}(0)<0 \text { and } v(x)<0 \text { for } 0<x \leq \xi\right. \\
\text { and } v(x)=0 \text { for } x>\xi\}
\end{gathered}
$$

and this observation finally enables us to adapt classical arguments. A similar strategy has been used in Burger et al. (2014), which combines a variant of the operator $\mathcal{A}_{\xi}$ with tailor-made nonlinear fixed point arguments to prove the existence (but not the uniqueness) of unimodal and compactly supported solutions $\varrho$ to the stationary variant of (4). The involved linear Krein-Rutman argument is that $v=\varrho^{\prime}$ satisfies the linearized equation $g^{\prime}(\varrho) v=a * v$ on the compact support of $\varrho$.

Proposition 1 (Variant of the Krein-Rutman theorem) For any $0<\xi<\infty$, the largest eigenvalue $\lambda_{\xi}$ of $\mathcal{A}_{\xi}$ in $L_{\text {odd }}^{2}(\mathbb{R})$ is simple and the corresponding eigenspace is spanned by a unique eigenfunction $v_{\xi} \in \widetilde{\mathcal{N}}_{\xi}$ with $\left\|v_{\xi}\right\|_{2}=1$.

Proof Variational setting The operator $\mathcal{A}_{\xi}$ admits only real eigenvalues thanks to Lemma 1. It is also the Gâteaux derivative of the functional

$$
\mathcal{F}_{\xi}(v):=\frac{1}{2}\left\langle v, \mathcal{A}_{\xi} v\right\rangle=\frac{1}{2}\langle\chi \xi \cdot v, a *(\chi \xi \cdot v)\rangle,
$$

which is well-defined on $\mathrm{L}_{\text {odd }}^{2}(\mathbb{R})$ since Young's inequality implies

$$
2\left|\mathcal{F}_{\xi}(v)\right| \leq\left\|\chi_{\xi} \cdot v\right\|_{2}\|a *(\chi \xi \cdot v)\|_{2} \leq\|a\|_{1}\left\|\chi_{\xi}\right\|_{\infty}^{2}\|v\|_{2}^{2} \leq\|v\|_{2}^{2}
$$

Moreover, the largest eigenvalue $\lambda_{\xi}$ of $\mathcal{A}_{\xi}$ can be characterized variationally via

$$
\lambda_{\xi}=\max \left\{2 \mathcal{F}_{\xi}(v): v \in \mathrm{L}_{\text {odd }}^{2}(\mathbb{R}),\|v\|_{2}=1\right\}
$$

where any maximizer corresponds to a normalized $\mathrm{L}_{\text {odd }}^{2}$-eigenfunction to $\lambda_{\xi}$ and vice versa.

Existence of eigenfunctions in $\mathcal{N}$ Any $v \in \mathrm{L}_{\text {odd }}^{2}(\mathbb{R})$ admits a unique and disjoint-support splitting

$$
v=v_{-}-v_{+} \quad \text { with } \quad v_{-}, v_{+} \in \mathcal{N} \text { and }\|v\|_{2}^{2}=\left\|v_{-}\right\|_{2}^{2}+\left\|v_{+}\right\|_{2}^{2},
$$

and using Assumption 1, Lemma 1, as well as (18) we verify

$$
\begin{aligned}
\mathcal{F}_{\xi}(v) & =\frac{1}{2}\left\langle v_{-}, \mathcal{A}_{\xi} v_{-}\right\rangle+\frac{1}{2}\left\langle v_{+}, \mathcal{A}_{\xi} v_{+}\right\rangle-\left\langle v_{-}, \mathcal{A}_{\xi} v_{+}\right\rangle \\
& \leq \mathcal{F}_{\xi}\left(v_{+}\right)+\mathcal{F}_{\xi}\left(v_{-}\right)
\end{aligned}
$$


Now suppose that $v \in \mathrm{L}_{\text {odd }}^{2}(\mathbb{R})$ is a maximizer for (22) with $v_{-} \neq 0$ and $v_{+} \neq 0$. We then have

$$
\mathcal{F}_{\xi}\left(v_{-} /\left\|v_{-}\right\|_{2}\right) \leq \mathcal{F}_{\xi}(v), \quad \mathcal{F}_{\xi}\left(v_{+} /\left\|v_{+}\right\|_{2}\right) \leq \mathcal{F}_{\xi}(v)
$$

while the homogeneity of $\mathcal{F}_{\xi}$ along with (24) yields

$$
\mathcal{F}_{\xi}(v) \leq\left\|v_{-}\right\|_{2}^{2} \mathcal{F}_{\xi}\left(v_{-} /\left\|v_{-}\right\|_{2}\right)+\left\|v_{+}\right\|_{2}^{2} \mathcal{F}_{\xi}\left(v_{+} /\left\|v_{+}\right\|_{2}\right)
$$

The combination of the two latter results with (23) and the normalization condition $\|v\|_{2}^{2}=1$ implies equality in both parts of (25). We conclude that $v_{-}$and $v_{+}$are eigenfunctions to the maximal eigenvalue $\lambda_{\xi}$ that belong to $\mathcal{N}$.

Regularity by refined cone analysis We next show that any eigenfunction $v \in \mathcal{N}$ to $\lambda_{\xi}$ belongs in fact to the smaller cone $\tilde{\mathcal{N}}_{\xi}$. The strict unimodality and the evenness of $a$ imply

$$
a(x-y)-a(x+y)>0 \text { for all } 0<x, y<\xi
$$

so (20) guarantees $\left(\mathcal{A}_{\xi} v\right)(x)<0$ and hence $v(x)<0$ for all $0<x \leq \xi$, where the first strict order relation can be inferred from $\chi \xi v \neq 0$. Moreover, we have

$$
\begin{aligned}
& \left(\mathcal{A}_{\xi} v\right)^{\prime}(x)=\int_{0}^{\xi}\left(a^{\prime}(x-y)-a^{\prime}(x+y)\right) v(y) \mathrm{d} y \\
& \left(\mathcal{A}_{\xi} v\right)^{\prime}(0)=-2 \int_{0}^{\xi} a^{\prime}(y) v(y) \mathrm{d} y<0
\end{aligned}
$$

and obtain $v \in \mathrm{BC}^{1}\left(I_{\xi}\right)$ with $v^{\prime}(0)<0$. Here we used $\lambda \xi>0$, which holds because (20) and (26) provide $\mathcal{F}_{\xi}(v)>0$ for any nontrivial $v \in \mathcal{N}$.

Uniqueness of normalized eigenfunctions We first prove the uniqueness within $\mathcal{N}$ as follows. Any two normalized eigenfunctions $v_{1}$ and $v_{2}$ to $\lambda_{\xi}$ lie in $\widetilde{\mathcal{N}}_{\xi}$ and the properties of this cone imply the existence of a parameter $0<s<\infty$ such that $v_{1}-s v_{2}$ still belongs to $\mathcal{N}$ but no longer to $\widetilde{\mathcal{N}}_{\xi}$ (because of $v_{1}^{\prime}(0)=s v_{2}^{\prime}(0)$ or $v_{1}(x)=s v_{2}(x)$ for at least one $\left.0<x \leq \xi\right)$. This, however, means $s=1$ as well as $v_{1}=v_{2}$ because otherwise $v_{1}-s v_{2}$ would be nontrivial eigenfunction in $\mathcal{N}$ that violates the above regularity result. Secondly, suppose there exists a nontrivial and normalized eigenfunction $v$ that belongs to neither $\mathcal{N}$ or $-\mathcal{N}$. Our results derived so far imply that $v_{-} /\left\|v_{-}\right\|$and $v_{+} /\left\|v_{+}\right\|$are identically maximizers of (22) and hence a contradiction to the assumption on $v$. In summary, we have shown that the eigenspace to $\lambda_{\xi}$ in $\mathrm{L}_{\text {odd }}^{2}(\mathbb{R})$ is one-dimensional and spanned by a unique normalized function $v_{\xi} \in \tilde{\mathcal{N}}_{\xi}$. 

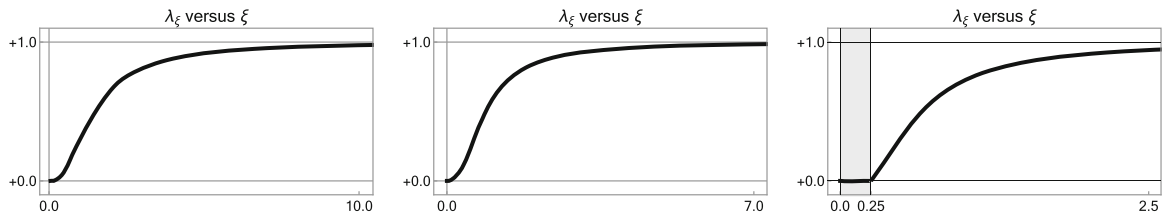

Fig. 5 Numerical values of the Krein-Rutman eigenvalue $\lambda \xi$ from Proposition 2 for the kernels (9) (left), (10) (center), and (11) (right). Notice the constant region in the last example (gray box), which stems from the constant plateau in the kernel

\section{Parameter Dependence}

The second building block for our nonlinear uniqueness result in Sect. 3 is the following properties of the Krein-Rutman quantities. Related numerical simulations are presented in Fig. 5.

Proposition 2 ( $\xi$-dependence of $\lambda_{\xi}$ and $v_{\xi}$ ) The map $\xi \mapsto \lambda_{\xi}$ from Proposition 1 is continuous and strictly increasing with

$$
\lim _{\xi \searrow 0} \lambda_{\xi}=0, \quad \lim _{\xi \nearrow \infty} \lambda_{\xi}=1
$$

Moreover, the corresponding map $\xi \mapsto v_{\xi}$ is $L^{2}$-continuous.

Proof Monotonicity Let $0<\xi_{1}<\xi_{2}<\infty$ be fixed. Since $v_{\xi_{1}}$ from Proposition 1 is supported in $I_{\xi_{1}}$, we have

$$
\chi \xi_{1} \cdot v_{\xi_{1}}=v_{\xi_{1}}=\chi \xi_{2} \cdot v_{\xi_{1}}, \quad \mathcal{F}_{\xi_{1}}\left(v_{\xi_{1}}\right)=\mathcal{F}_{\xi_{2}}\left(v_{\xi_{1}}\right)
$$

and the optimization problem (22) ensures the nonstrict monotonicity $\lambda \xi_{1} \leq \lambda \xi_{2}$. However, $v_{\xi_{1}}$ cannot be an eigenfunction of $\mathcal{A}_{\xi_{2}}$ because the properties of $a$ imply that $\mathcal{A}_{\xi_{2}} v_{\xi_{1}}$ has a larger support than $v_{\xi_{1}} \in \widetilde{\mathcal{N}}_{\xi_{1}}$. We thus obtain $\lambda \xi_{1} \neq \lambda \xi_{2}$.

Continuity and asymptotics for small $\xi$ Suppose that $\left(\xi_{n}\right)_{n \in \mathbb{N}}$ is a given sequence with

$$
\xi_{n} \rightarrow \xi \in(0, \infty), \quad \lambda \xi_{n} \rightarrow \lambda \in(0, \infty)
$$

By weak compactness we can extract a (not relabeled) subsequence such that the normalized eigenfunctions $v_{\xi_{n}}$ converge weakly to some limit $v \in \mathrm{L}_{\text {odd }}^{2}(\mathbb{R})$, where the weak closedness of $\mathcal{N}$ ensures $v \in \mathcal{N}$. The properties of convolution operators combined with $\chi_{\xi_{n}} \rightarrow \chi_{\xi}$ in $\mathrm{L}^{2}(\mathbb{R})$ imply that $\mathcal{A}_{\xi_{n}} v_{\xi_{n}}$ converges strongly to $\mathcal{A}_{\xi} v$ and this guarantees - thanks to the eigenvalue equation for $v_{\xi_{n}}$ - the strong convergence of $v_{\xi_{n}}$. In particular, we obtain

$$
\lambda v=\mathcal{A}_{\xi} v, \quad\|v\|_{2}=1, \quad 2 \mathcal{F}_{\xi}(v)=\lambda
$$


and hence $\lambda \leq \lambda_{\xi}$. On the other hand, (22) ensures $\lambda_{\xi} \leq \lambda$ since

$$
2 \mathcal{F}_{\xi}(\tilde{v})=\lim _{n \rightarrow \infty} 2 \mathcal{F}_{\xi_{n}}(\tilde{v}) \leq \lim _{n \rightarrow \infty} \lambda \xi_{n}=\lambda
$$

holds for any $\tilde{v} \in \mathrm{L}_{\text {odd }}^{2}(\mathbb{R})$. We thus get $\lambda=\lambda_{\xi}$ and Proposition 1 combined with (27) yields $v=v_{\xi}$. In summary, we have shown for any convergent sequence $\left(\xi_{n}\right)_{n \in \mathbb{N}}$ that $\lambda_{\xi}$ is the unique accumulation point of the sequence $\left(\lambda_{\xi_{n}}\right)_{n \in \mathbb{N}}$ (which is bounded due to the monotonicity with respect to $\xi$ ), and this gives rise to the claimed continuity of $\lambda_{\xi}$. Our arguments also yield the strong convergence $v_{\xi_{n}} \rightarrow v_{\xi}$, and analogously we derive $\xi_{n} \rightarrow 0$ from $\lambda \xi_{n} \rightarrow 0$ because $\mathcal{A}_{\xi_{n}} v_{\xi_{n}} \rightarrow 0$ holds along any weakly convergent subsequence.

Asymptotics for large $\xi$ Direct computations for the piecewise constant and compactly supported test function $\tilde{v}_{\xi}$ with

$$
\tilde{v}_{\xi}(x)=-(2 \xi)^{-1 / 2} \operatorname{sgn}(x) \chi \xi(x)
$$

yield $\left\|\tilde{v}_{\xi}\right\|_{2}=1$ as well as

$$
\begin{aligned}
\mathcal{F}_{\xi}\left(\tilde{v}_{\xi}\right) & =\frac{1}{4 \xi} \int_{-\xi}^{+\xi} \int_{-\xi}^{+\xi} a(x-y) \operatorname{sgn}(x) \operatorname{sgn}(y) \mathrm{d} y \mathrm{~d} x \\
& =\frac{1}{2 \xi} \int_{0}^{\xi} \int_{0}^{\xi}(a(x-y)-a(x+y)) \mathrm{d} y \mathrm{~d} x \\
& =\int_{0}^{\xi} a(z) \mathrm{d} z-\frac{3}{2} \int_{0}^{\xi} \frac{z}{\xi} a(z) \mathrm{d} z-\frac{1}{2} \int_{\xi}^{2 \xi} \frac{(2 \xi-z)}{\xi} a(z) \mathrm{d} z
\end{aligned}
$$

where the last identity holds due to elementary splitting arguments and integral transformations.

Using (22) and the Dominated Convergence Theorem, we therefore get

$$
\liminf _{\xi \rightarrow \infty} \lambda_{\xi} \geq 2 \lim _{\xi \rightarrow \infty} \mathcal{F}_{\xi}\left(\tilde{v}_{\xi}\right)=2 \int_{0}^{\infty} a(z) \mathrm{d} z=1
$$

thanks to Assumption 1. On the other hand, the Young estimate (21) applied to $v_{\xi}$ ensures

$$
\limsup _{\xi \rightarrow \infty} \lambda_{\xi}=2 \limsup _{\xi \rightarrow \infty} \mathcal{F}_{\xi}\left(v_{\xi}\right) \leq 1
$$

and the proof is complete. 


\section{Approximation}

The unique normalized Krein-Rutman eigenfunction as provided by Proposition 2 can be computed as the limit of the sequence $\left(v_{n}\right)_{n \in \mathbb{N}} \subset \mathcal{N}$ with

$$
v_{n}:=\frac{\mathcal{A}_{\xi}^{n} v_{0}}{\left\|\mathcal{A}_{\xi}^{n} v_{0}\right\|_{2}}
$$

and arbitrary $v_{0} \in \mathcal{N}$. Moreover, since $\mathcal{F}_{\xi}(-\operatorname{sgn}|v|) \geq \mathcal{F}_{\xi}(v)$ holds for any $v \in$ $\mathrm{L}_{\text {odd }}^{2}(\mathbb{R})$, we deduce that $\lambda \xi$ is not only the largest eigenvalue but also the spectral radius of $\mathcal{A}_{\xi}$ and hence the exponential convergence of $v_{n}$ as $n \rightarrow \infty$. This approximation scheme is often called Power Method, and a straightforward discretization (fine but equidistant spatial grid and Riemann sums instead of integrals) has been used to produce the numerical results in this paper. Alternatively, one can write the sequence as

$$
\lambda_{n} v_{n+1}=\mathcal{A}_{\xi} v_{n}, \quad \lambda_{n}=\left\|\mathcal{A}_{\xi} v_{n}\right\|_{2},
$$

where $\lambda_{n}$ will converge as $n \rightarrow \infty$ to the Krein-Rutman eigenvalue $\lambda \xi$. A similar improvement dynamics can also be applied to the nonlinear eigenvalue problem (1), but the convergence is more subtle due to the lack of uniqueness results for general functions $f$. We refer to Herrmann and Matthies (2020) for numerical examples and a more detailed discussion of the analytical properties.

\section{Generalizations}

We finally discuss the case of less regular kernels $a$. The crucial ingredient to the regularity step $v \in \mathcal{N} \Rightarrow \mathcal{A}_{\xi} v \in \widetilde{\mathcal{N}}_{\xi}$ in the proof of Proposition 1 is (26). This condition hinges on the strict unimodality of $a$ as in (8) and is not satisfied for all $a \in \mathcal{U}$, see Fig. 6 for an illustration. For kernels like the tent map (10), a recursive argument reveals the implication $v \in \mathcal{N} \Rightarrow \mathcal{A}_{\xi}^{n} v \in \widetilde{\mathcal{N}}_{\xi}$ for all sufficiently large $n \in \mathbb{N}$, and this guarantees that all assertions in Proposition 1 remain valid since the Krein-Rutman eigenfunction still belongs to $\tilde{\mathcal{N}}_{\xi}$. This, however, is no longer true for the kernel (11). Instead, we have to distinguish between the following three parameter regimes:

1. $0<\xi \leq \frac{1}{4}$ implies that $\mathcal{A}_{\xi} v$ vanishes for any $v \in \mathrm{L}_{\text {odd }}^{2}(\mathbb{R})$, so $\mathcal{A}_{\xi}$ restricted to $\mathrm{L}_{\text {odd }}^{2}(\mathbb{R})$ is actually the trivial operator and we have $\lambda_{\xi}=0$, see the third panel in Fig. 5.

2. In the case of $\frac{1}{4}<\xi<\frac{1}{2}$, there exists a unique and normalized Krein-Rutman eigenfunction $v_{\xi}$, which does not belong to $\tilde{\mathcal{N}}_{\xi}$ but to a modified subcone of $\mathcal{N}$ with

$$
\lim _{x \searrow \frac{1}{2}-\xi} v^{\prime}(x)<0
$$


Fig. 6 Support of the integral kernel corresponding to $\mathcal{A}_{\xi}$, see (20), for several values of $\xi$ and the degenerate convolution kernels (10) (top row) and (11) (bottom row). Since condition (26) is not satisfied for some values of $\xi$, the proof and/or the precise statement of

Proposition 1 needs to be modified as discussed in the text. See also Fig. 5 for the corresponding Krein-Rutman eigenvalues
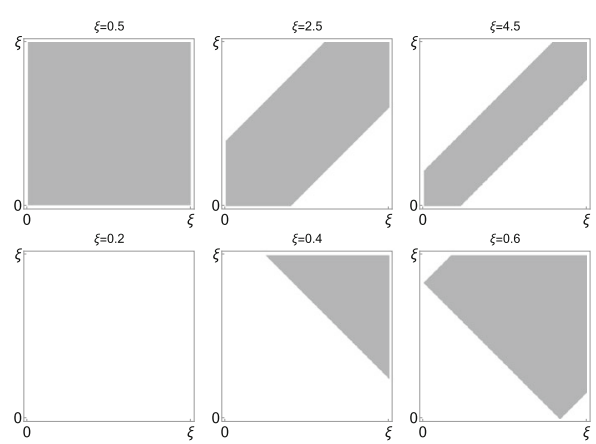

$\xi=0.4$

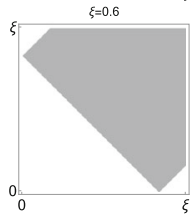

and

$$
v(x)<0 \text { for } \frac{1}{2}-\xi<x \leq \xi, \quad v(x)=0 \text { for } 0<x<\frac{1}{2}-\xi \text { and } x>\xi,
$$

see the first column in Fig. 4.

3. For $\xi \geq \frac{1}{2}$, we find again $v_{\xi} \in \tilde{\mathcal{N}}_{\xi}$.

In summary, if $a$ is given by (11), then Proposition 1 must be formulated more carefully and the analogue to Proposition 2 stipulates the one-to-one correspondence between $\xi \in\left(\frac{1}{4}, \infty\right)$ and $\lambda_{\xi} \in(0,1)$. Similar statements apply to any other kernel $a \in \mathcal{U}$ that admits a constant plateau near $x=0$.

\section{Comments}

The linear eigenvalue problem (17) has also been studied in Burger et al. (2013) in the context of biological aggregation models. More precisely, the stationary variant of (4) with linear constitutive function $g(r)=\lambda r$ can be written as

$$
\varrho \partial_{x}(\lambda \varrho-a * \varrho)=0
$$

and assuming the compact support condition

$$
\operatorname{supp} \varrho=[-\xi,+\xi]
$$

we readily show under natural regularity assumptions on $\varrho$ that $v=\varrho^{\prime}$ must be an eigenfunction of $\mathcal{A}_{\xi}$ with eigenvalue $\lambda$. According to [Burger et al. (2013), Propositions 4.11 and 4.12], there exists a unique one-parameter family of solutions $\varrho \in \mathcal{U}$ to (28) and (29) which can be parametrized by $\lambda \in(0,1)$ or $\xi \in(0, \infty)$. The corresponding proofs share many similarities with our line of argument since they are likewise based on both the strict unimodality of the convolution kernel and the Krein-Rutman theorem applied to (17). The setting and the discussion of the technical details, however, are different and rely on other cones and estimates. Moreover, the nonlinear eigenfunctions in our setting are not compactly supported but decay smoothly at infinity. 


\section{Nonlinear Eigenfunctions with Unimodal Profile}

We are now able to prove our main result from Sect. 1 in two steps. First, we show that the general case with given kernel $a$ and $\zeta>0$ is equivalent to the special case $\zeta=0$ for a modified kernel $\tilde{a}$.

Proposition 3 (transformation to special case) A function $u$ solves (1) with $\sigma \in$ $(\zeta, \zeta+\eta)$ if and only if the eigenvalue equation

$$
\tilde{\sigma} u=\tilde{a} * \tilde{f}(u)
$$

is satisfied with

$$
\tilde{\sigma}:=\sigma-\zeta=(1-\mu) \sigma \in(0, \eta), \quad \mu:=\sigma^{-1} \zeta \in(0,1)
$$

Here, the transformed kernel

$$
\tilde{a}:=(1-\mu) a *\left(1+\mu a+\mu^{2} a * a+\mu^{3} a * a * a+\ldots\right),
$$

is well-defined, depends on $\sigma$, and complies with Assumption 1, while the simplified nonlinearity

$$
\tilde{f}(r):= \begin{cases}0 & \text { for } r \in[0, \theta], \\ \eta(r-\theta) & \text { for } r \in[\theta, \infty),\end{cases}
$$

represents (6) with $\zeta=0$.

Proof Linear auxiliary operator and modified kernel The Young estimate

$$
\|a * w\|_{2} \leq\|a\|_{1}\|w\|_{2}=\|w\|_{2}
$$

combined with $0<\mu<1$ implies that the linear operator

$$
\mathcal{L}: \mathrm{L}^{2}(\mathbb{R}) \rightarrow \mathrm{L}^{2}(\mathbb{R}), \quad \mathcal{L} w:=w-\mu a * w
$$

is continuously invertible. The Neumann formula

$$
\mathcal{L}^{-1} w=\breve{a} * w, \quad \breve{a}:=1+\mu a+\mu^{2} a * a+\mu^{3} a * a * a+\ldots
$$

reveals $\breve{a} \in \mathcal{U}$ thanks to Lemma 1 , which in turn ensures that $\mathcal{L}$ respects the even-odd parity and mediates the implication (19). We further have

$$
\tilde{a}=(1-\mu) a * \breve{a}=(1-\mu) \breve{a} * a \in \mathcal{U}
$$


and compute its mass

$$
\int_{\mathbb{R}} \tilde{a}(x) \mathrm{d} x=(1-\mu) \sum_{k=0}^{\infty} \mu^{k}=1
$$

as well as the derivative

$$
\tilde{a}^{\prime}:=(1-\mu) \breve{a} * a^{\prime} .
$$

The latter formula provides via

$$
\tilde{a}^{\prime}(x)<0 \text { for all } x>0
$$

the strict unimodality of $\tilde{a}$.

Transformation of the nonlinear problem Let $(\sigma, u)$ be a given solution to (1). Our definitions imply

$$
\begin{aligned}
\tilde{\sigma}(u-\mu a * u) & =(1-\mu)(\sigma u-\zeta a * u)=(1-\mu)(a *(f(u)-\zeta u)) \\
& =(1-\mu) a * \tilde{f}(u)
\end{aligned}
$$

and hence the validity of (30) thanks to the existence of $\mathcal{L}^{-1}$. Similarly, the reverse implication follows by applying $\mathcal{L}$ to both sides of (30).

In the second step we finally establish our existence and uniqueness result for nonlinear eigenfunctions.

Proposition 4 (Nonlinear existence and uniqueness result) Equation (1) admits for any $\sigma \in(\zeta, \zeta+\eta)$ a unique solution $u_{\sigma} \in \mathcal{U}$.

Proof Preliminaries Within this proof, $\sigma$ is fixed. In view of Proposition 3, we can assume $\zeta=0$ with $\sigma \in(0, \eta)$ because otherwise we replace $a$ by $\tilde{a}$ and $\sigma$ by $\tilde{\sigma}$. Moreover, Proposition 2 guarantees that

$$
\lambda \xi_{\sigma}=\eta^{-1} \sigma
$$

holds for precisely one cut-off parameter $\xi_{\sigma} \in(0, \infty)$.

Existence and construction By integration, there exists a unique function $\tilde{u}_{\sigma} \in \mathcal{U}$ with

$$
\tilde{u}_{\sigma}^{\prime}(x)=v_{\xi_{\sigma}}(x) \text { for } x \in \mathbb{R}, \quad \tilde{u}_{\sigma}(x)=0 \text { for }|x| \geq \xi_{\sigma},
$$

where $v_{\xi_{\sigma}} \in \widetilde{\mathcal{N}}_{\xi}$ is the normalized and compactly supported Krein-Rutman eigenfunction from Proposition 1. Since $\tilde{u}_{\sigma}$ is strictly positive for $|x|<\xi_{\sigma}$, the function $a * \tilde{u}_{\sigma}$ attains a positive value at $x=\xi_{\sigma}$. We thus define $u_{\sigma} \in \mathcal{U}$ by

$$
u_{\sigma}:=\tau_{\sigma} a * \tilde{u}_{\sigma}, \quad \tau_{\sigma}:=\frac{\theta}{\left(a * \tilde{u}_{\sigma}\right)\left(\xi_{\sigma}\right)}
$$


and observe that this guarantees

$$
u_{\sigma}\left( \pm \xi_{\sigma}\right)=\theta, \quad \theta \leq u_{\sigma}(x) \text { for }|x|<\xi_{\sigma}, \quad 0 \leq u_{\sigma}(x) \leq \theta \text { for }|x|>\xi_{\sigma}
$$

due to the unimodality of $u_{\sigma}$. Since we also have

$$
u_{\sigma}^{\prime}(x)=\tau_{\sigma}\left(a * \tilde{u}_{\sigma}^{\prime}\right)(x)=\tau_{\sigma}\left(a * v_{\xi_{\sigma}}\right)(x)=\tau_{\sigma} \lambda_{\xi_{\sigma}} v_{\xi_{\sigma}}(x)=\tau_{\sigma} \lambda_{\xi_{\sigma}} \tilde{u}_{\sigma}^{\prime}(x)
$$

for all $|x| \leq \xi_{\sigma}$ (but not for $|x|>\xi_{\sigma}$ ), we find

$$
u_{\sigma}(x)=\theta+\tau_{\sigma} \lambda \xi_{\sigma} \tilde{u}_{\sigma}(x) \text { for }|x| \leq \xi_{\sigma}
$$

In particular, we have

$$
f\left(u_{\sigma}(x)\right)=\tau_{\sigma} \lambda \xi_{\sigma} \eta \tilde{u}_{\sigma}(x) \text { for all } x \in \mathbb{R}
$$

thanks to (6) with $\zeta=0$, so

$$
a * f\left(u_{\sigma}\right)=a *\left(\tau_{\sigma} \lambda \xi_{\sigma} \eta \tilde{u}_{\sigma}\right)=\lambda \xi_{\sigma} \eta u_{\sigma}=\sigma u_{\sigma}
$$

follows from our definition of $u_{\sigma}$ in (34).

Uniqueness Now suppose that $0 \neq u \in \mathcal{U}$ solves (1) for $\zeta=0$. This gives $0<$ $u(0)=\|u\|_{\infty}$ (otherwise $f(u)$ would vanish identically) and there exists $\xi \in(0, \infty)$ with $u(\xi)=\theta$. By differentiating (1) with respect to $x$-and using the notations (15), (16) — we establish the formulas (14) and (17), so Propositions 1 and 2 provide

$$
\xi=\xi_{\sigma} \quad \text { and } \quad v=c v_{\xi_{\sigma}}
$$

for some factor $c>0$. Combining this with (33) and (34) we conclude that the derivatives of $u$ and $u_{\sigma}$ are proportional, and the consistency relations

$$
-\int_{\xi_{\sigma}}^{\infty} u^{\prime}(x) \mathrm{d} x=u\left(\xi_{\sigma}\right)=\theta=u_{\sigma}\left(\xi_{\sigma}\right)=-\int_{\xi_{\sigma}}^{\infty} u_{\sigma}^{\prime}(x) \mathrm{d} x
$$

give $u=u_{\sigma}$.

\section{Comments}

Notice that the transformed kernel $\tilde{a}$ in (31) depends also on $\zeta$ and satisfies the analogue to the crucial condition (26) even if the kernel $a$ is not strictly unimodal due to a compact support or constant plateaus, see Fig. 7 for an illustration. Consequently, all results in this section cover for $\zeta>0$ the kernels (10) and (11) as well, and our comments at the end of Sect. 2 on how to generalize Propositions 1 and 2 are relevant for $\zeta=0$ only. 

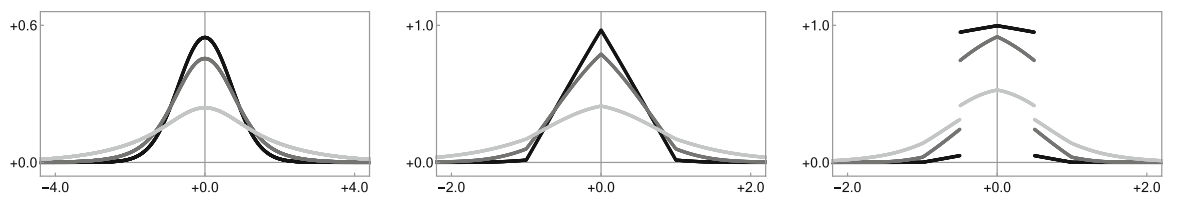

Fig. 7 The transformed kernel (31) for the Gaussian (left), the tent map (middle), and the indicator function (right) from (9), (10), and (11). The three curves correspond to $\mu=0.1$ (black), $\mu=0.5$ (dark gray), $\mu=0.9$ (light gray)

The transformed kernel $\tilde{a}$ also features prominently in Truskinovsky and Vainchtein (2014), which studies the bilinear eigenvalue problem for the tent map kernel by different methods. In our notations, the main ideas can be described as follows. The function $\tilde{w}$ with

$$
\tilde{w}(x)=\frac{\tilde{f}(u(x))}{\tilde{\sigma}}=\frac{\eta \chi \xi(x)(u(x)-\theta)}{\sigma-\zeta},
$$

represents the inhomogeneity, is continuous (especially at $x= \pm \xi$ ), and satisfies

$$
\tilde{w}^{\prime}(x)=\frac{\eta \chi_{\xi}(x) u^{\prime}(x)}{\sigma-\zeta}=\frac{\eta v(x)}{\sigma-\zeta}
$$

for all $x \in \mathbb{R}$. The linear problem for the effective derivative profile (15) can hence be written analogously to (17) as

$$
v(x)-\frac{\eta}{\sigma-\zeta} \int_{-\xi}^{+\xi} \tilde{a}(x-y) v(y) \mathrm{d} y=0 \text { for }-\xi \leq x \leq+\xi
$$

and Truskinovsky and Vainchtein (2014) solve this equation under the consistency relation

$$
\int_{0}^{\xi} v(x) \mathrm{d} x=\theta-u(0), \quad \int_{0}^{\xi}(1+x) v(x) \mathrm{d} x=\theta \xi
$$

by combining two ingredients. The fixed point problem (35) is solved with parameter $\xi$ by Wiener-Hopf methods. This requires detailed information on the Fourier transform of $\tilde{a}$ and direct computations reveal

$$
\widehat{\tilde{a}}(k)=\frac{(1-\mu) \widehat{a}(k)}{1-\mu \widehat{a}(k)}=\frac{\tilde{\sigma} \widehat{a}(k)}{\sigma-\zeta \widehat{a}(k)}=\frac{\tilde{\sigma}}{\eta}\left(1-\frac{(\zeta+\eta) \widehat{a}(k) k^{2}-\sigma k^{2}}{\zeta \widehat{a}(k) k^{2}-\sigma k^{2}}\right)
$$

as well as $\widehat{a}(k) k^{2}=4 \sin ^{2}(k / 2)$ for the kernel (10). Moreover, the value of $\xi$ is determined numerically by means of a scalar nonlinear equation equivalent to (32). This approach works well since $\widehat{a}$ is a nice known function and provides power series 
expressions for $v$ and $u$, which can be used to derive intricate but almost explicit approximation formulas.

We finally mention that nonnegative eigenfunctions (being unimodal or not) cannot exist for $\sigma \geq \zeta+\eta$ since (1) implies

$$
\sigma\|u\|_{2} \leq\|a * f(u)\|_{2} \leq\|a\|_{1}\|f(u)\|_{2} \leq(\zeta+\eta)\|u\|_{2},
$$

where the last estimate is actually strict for any nontrivial $u$. Similarly, using

$$
\sigma \int_{\mathbb{R}} u(x) \mathrm{d} x=\int_{\mathbb{R}} f(u(x)) \mathrm{d} x
$$

we can disprove the existence of nonnegative eigenfunctions for $\sigma \leq \zeta$ under the additional assumption $u \in \mathrm{L}^{1}(\mathbb{R})$. Notice that the eigenfunction from Proposition 4 is integrable due to (30) and because $\tilde{f}\left(u_{\sigma}\right)$ is always compactly supported. More precisely, $u_{\sigma}$ decays as fast as $a$ or the transformed kernel $\tilde{a}$ for $\zeta=0$ and $\zeta>0$, respectively. A similar argument has been applied in Herrmann and Matthies (2020), which proves the existence and the localization (but not the uniqueness) of nonlinear eigenfunctions for more general functions $f$ in a nonlinear variational setting.

Funding Open Access funding enabled and organized by Projekt DEAL.

\section{Compliance with Ethical Standards}

Conflict of interest The authors declare that they have no conflict of interest.

Open Access This article is licensed under a Creative Commons Attribution 4.0 International License, which permits use, sharing, adaptation, distribution and reproduction in any medium or format, as long as you give appropriate credit to the original author(s) and the source, provide a link to the Creative Commons licence, and indicate if changes were made. The images or other third party material in this article are included in the article's Creative Commons licence, unless indicated otherwise in a credit line to the material. If material is not included in the article's Creative Commons licence and your intended use is not permitted by statutory regulation or exceeds the permitted use, you will need to obtain permission directly from the copyright holder. To view a copy of this licence, visit http://creativecommons.org/licenses/by/4.0/.

\section{Appendix: Formal Asymptotic Analysis}

We characterize the limiting behavior of the nonlinear eigenfunction $u_{\sigma}$ from Proposition 4 . We always start with the special case $\zeta=0$ and discuss the necessary modifications in the case $\zeta>0$ afterward. The natural quantity for the formal asymptotic analysis with $\zeta=0$ is the compactly supported functions

$$
w_{\sigma}(x):=\sigma^{-1} f\left(u_{\sigma}(x)\right),
$$


which determine $u_{\sigma}$ via

$$
u_{\sigma}=a * w_{\sigma}
$$

We rescale $w_{\sigma}$ according to

$$
\bar{w}_{\sigma}(\bar{x})=w(x), \quad x=\xi_{\sigma} \bar{x},
$$

because this allows us to work on the fixed interval $\bar{I}:=[-1,+1]$, and notice that

$$
\bar{w}_{\sigma}( \pm 1)=0 \text {. }
$$

Moreover, the nonlinear eigenvalue equation (1) combined with

$$
\chi \xi_{\sigma} u_{\sigma}=\theta \chi_{\xi_{\sigma}}+\lambda_{\sigma} w_{\sigma}, \quad \sigma=\eta \lambda_{\sigma}
$$

yields

$$
\theta+\lambda_{\sigma} \bar{w}_{\sigma}=\overline{\mathcal{A}}_{\xi_{\sigma}} \bar{w}_{\sigma} \text { for } \bar{x} \in \bar{I}
$$

where the operator

$$
\left(\overline{\mathcal{A}}_{\xi} \bar{w}\right)(\bar{x}):=\int_{-1}^{+1} \xi a(\xi \bar{x}-\xi \bar{y}) \bar{w}(\bar{y}) \mathrm{d} \bar{y}
$$

is the rescaled counterpart of $\mathcal{A}_{\xi}$ from (18).

\section{Small Eigenvalues for $\zeta=0$}

Proposition 2 shows $\xi_{\sigma} \approx 0$, and by Taylor expansion of (39) we get

$$
\begin{aligned}
\left(\overline{\mathcal{A}}_{\xi} \bar{w}\right)(\bar{x})=\xi & \cdot\left(a(0) \int_{-1}^{+1} \bar{w}(\bar{y}) \mathrm{d} \bar{y}\right)-\xi^{3} \cdot\left(\frac{1}{2}\left|a^{\prime \prime}(0)\right| \int_{-1}^{+1} \bar{w}(\bar{y}) \bar{y}^{2} \mathrm{~d} \bar{y}\right) \\
& -\bar{x}^{2} \cdot \xi^{3} \cdot\left(\frac{1}{2}\left|a^{\prime \prime}(0)\right| \int_{-1}^{+1} \bar{w}(\bar{y}) \mathrm{d} \bar{y}\right)+O\left(\xi^{5}\right) .
\end{aligned}
$$

The eigenvalue equation (38) thus implies the approximate identity

$$
\bar{w}_{\sigma}(\bar{x}) \approx c_{\sigma}\left(1-\bar{x}^{2}\right)
$$



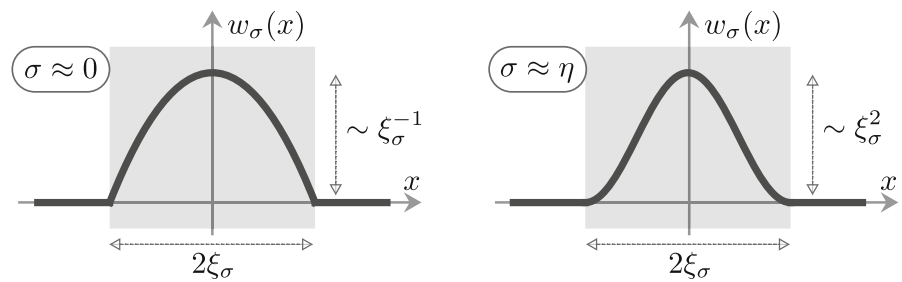

Fig. 8 Schematic representation of the approximate formulas (40) (left panel, $\xi_{\sigma} \sim \sigma^{1 / 3}$ ) and (46) (right panel, $\left.\xi_{\sigma} \sim(\eta-\sigma)^{-1}\right)$, which describe the asymptotics of $u_{\sigma}$ in the special case $\zeta=0$. See also (36) and (37) as well as Conjectures 1 and 3

for some constant $c_{\sigma}$ and any $\bar{x} \in[-1,+1]$. Computing the integrals, we find

$$
\begin{aligned}
\theta+\lambda_{\sigma} c_{\sigma}\left(1-\bar{x}^{2}\right) \approx & c_{\sigma} \xi_{\sigma}\left(\frac{4}{3} a(0)\right)-c_{\sigma} \xi_{\sigma}^{3}\left(\frac{2}{15}\left|a^{\prime \prime}(0)\right|\right) \\
& -\bar{x}^{2} c_{\sigma} \xi_{\sigma}^{3}\left(\frac{2}{3}\left|a^{\prime \prime}(0)\right|\right) .
\end{aligned}
$$

and equating the coefficients-first in front of $\bar{x}^{2}$ and afterward in front of 1 -we identify the scaling relations

$$
\lambda_{\sigma} \approx \frac{2}{3}\left|a^{\prime \prime}(0)\right| \xi_{\sigma}^{3}, \quad \frac{4}{3} a(0) c_{\sigma} \xi_{\sigma} \approx \theta
$$

where $\lambda_{\sigma}$ satisfies (32). Moreover, the function

$$
w_{\sigma}(x) \approx \frac{3 \theta}{4 a(0) \xi_{\sigma}} \chi_{\xi_{\sigma}}(x)\left(1-\xi_{\sigma}^{-2} x^{2}\right)
$$

approaches a Dirac distribution as $\sigma \rightarrow 0$, see also Fig. 8, so (37) implies that $u_{\sigma}$ converges to a multiple of $a$, where the scaling factor is consistent with $u_{\sigma}\left(\xi_{\sigma}\right)=\theta$. In summary, we expect the following behavior for small $\sigma$.

Conjecture 1 (Asymptotics for $\zeta=0$ and $\sigma \approx 0$ ) Suppose that the kernel a that is sufficiently smooth at the origin with $a^{\prime \prime}(0)<0$. Then, we have

$$
\sigma^{-1 / 3} \xi_{\sigma} \stackrel{\sigma \searrow 0}{\longrightarrow} \frac{3^{1 / 3}}{2^{1 / 3} \eta^{1 / 3}\left|a^{\prime \prime}(0)\right|^{1 / 3}}
$$

as well as

$$
\sigma^{1 / 3} \bar{w}_{\sigma}(\bar{x}) \stackrel{\sigma \searrow 0}{\longrightarrow} \frac{3^{2 / 3} \eta^{1 / 3} \theta\left|a^{\prime \prime}(0)\right|^{1 / 3}}{2^{5 / 3} a(0)} \bar{\chi}(\bar{x})\left(1-\bar{x}^{2}\right)
$$

where $\bar{\chi}$ is the indicator function of $\bar{I}$. Moreover,

$$
\lim _{\sigma \searrow 0} u_{\sigma}(x)=\frac{\theta a(x)}{a(0)}, \quad \lim _{\sigma \searrow 0} f\left(u_{\sigma}(x)\right)=0
$$



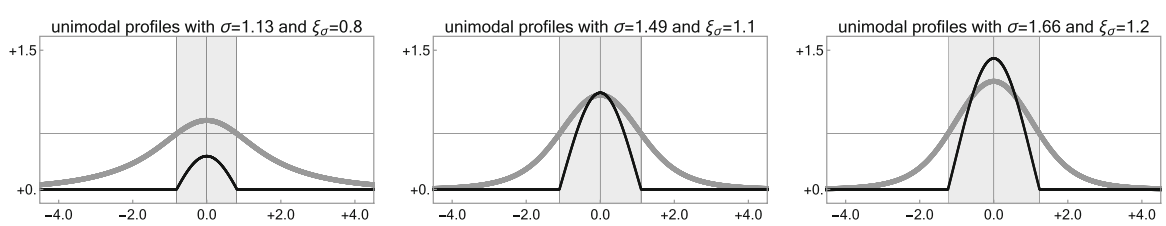

Fig. 9 Numerical simulation with $\zeta=1.0, \theta=0.6, \eta=2.5$ for the Gaussian kernel (9). The computations are performed with the nonlinear improvement dynamics described in Herrmann and Matthies (2020), and the profiles $u_{\sigma}$ (gray) and $\tilde{f}\left(u_{\sigma}\right)$ (black) are shown for several values of $\sigma$. Conjecture 2 predicts that $u_{\sigma}$ converges as $\sigma \searrow \zeta$ to the constant function with value $\theta$, but this limit is hard to capture numerically

\section{holds at least in the sense of pointwise convergence.}

We believe that the assertions of Conjecture 1 can be derived by standard arguments but notice that (41) and (42) do not cover the kernels (10) and (11) since these are not smooth and satisfy $a^{\prime \prime}(0)=-\infty$ and $a^{\prime \prime}(0)=0$, respectively. Nonetheless, a modified asymptotic analysis should reveal that (43) is still satisfied. For the tent map kernel, a similar convergence result has been derived in Truskinovsky and Vainchtein (2014) for $\zeta>0$ and $\eta \rightarrow \infty$. This limit has much in common with the anticontinuum or high-energy limit studied in Herrmann and Matthies (2019b).

\section{Small Eigenvalues for $\zeta>0$}

In this case, we have to replace the kernel $a$ by the transformed kernel from Proposition 3, which we now denote by $\tilde{a}_{\sigma}$ as it depends on $\sigma$, and $\sigma$ by $\tilde{\sigma}=\sigma-\zeta$. The problem is that $\tilde{a}_{\sigma}$ does not converge strongly as $\sigma \searrow \zeta$ but its amplitude gets smaller while its effective width approaches $\infty$, see Fig. 7 for an illustration. The precise analysis depends on the singular scaling behavior of $\tilde{a}_{\sigma}$. For sufficiently nice kernels we can suppose that the limits

$$
\kappa_{0}:=\lim _{\sigma \searrow \zeta}(\sigma-\zeta)^{-1 / 2} \tilde{a}_{\sigma}(0), \quad \kappa_{2}:=\lim _{\sigma \searrow \zeta}(\sigma-\zeta)^{-1}\left|\tilde{a}_{\sigma}^{\prime \prime}(0)\right|
$$

are well defined, and direct computations for the Gaussian kernel (9) provide the values $\kappa_{0}=\zeta^{-1 / 2}$ as well as $\kappa_{2}=2 \pi^{-1 / 2} \zeta^{-1} \operatorname{Zeta(3/2)}$ in terms of the Riemann zeta function and the parameter $\zeta$.

In consistency with Proposition 3, we base our analysis of

$$
\overline{\tilde{w}}_{\sigma}(\bar{x})=\tilde{w}_{\sigma}\left(\xi_{\sigma} \bar{x}\right), \quad \tilde{w}_{\sigma}(x)=\frac{\tilde{f}\left(u_{\sigma}(x)\right)}{\tilde{\sigma}}
$$

which solves an equation similar to (38). The scaling relations encoded by (44) still allow us to Taylor expand $\tilde{a}_{\sigma}$ even though the corresponding cut-off parameter $\xi_{\sigma}$ can no longer assumed to be small. In fact, repeating the arguments from above we find

$$
\overline{\tilde{w}}(\bar{x})=\tilde{c}_{\sigma}\left(1-\bar{x}^{2}\right)
$$


as well as

$$
\begin{aligned}
\theta+\eta^{-1}(\sigma-\zeta) \tilde{c}_{\sigma}\left(1-\bar{x}^{2}\right) \approx & \frac{4}{3} \kappa_{0} \tilde{c}_{\sigma} \xi_{\sigma}(\sigma-\zeta)^{1 / 2}-\frac{2}{15} \kappa_{2} \tilde{c}_{\sigma} \xi_{\sigma}^{3}(\sigma-\zeta) \\
& -\bar{x}^{2} \frac{2}{3} \kappa_{2} \tilde{c}_{\sigma} \xi_{\sigma}^{3}(\sigma-\zeta)
\end{aligned}
$$

Equating coefficients gives

$$
\frac{2}{3} \kappa_{2} \xi_{\sigma}^{3} \approx \eta^{-1}, \quad \frac{4}{3} \kappa_{0} \tilde{c}_{\sigma} \xi_{\sigma}(\sigma-\zeta)^{1 / 2} \approx \theta
$$

as well as

$$
(\sigma-\zeta)^{1 / 2} \tilde{w}_{\sigma} \approx \frac{3 \theta}{4 \kappa_{0} \xi_{\sigma}} \chi \xi(x)\left(1-\xi_{\sigma}^{-2} x^{2}\right)
$$

and in summary we find a limiting behavior that differs considerably from Conjecture 1 . In particular, $(\sigma-\zeta)^{1 / 2} \tilde{w}_{\sigma}$ converges to a piecewise smooth limit function with integral $\kappa_{0}^{-1} \theta$, whose convolution with the small and slowly varying function $\tilde{a}_{\sigma}$ approximates $u_{\sigma}$ up to a small prefactor. See also Fig. 9.

Conjecture 2 (Asymptotics for $\zeta>0$ and $\sigma \approx \zeta$ ) Suppose that the kernel $a$ is sufficiently regular so that (44) is satisfied. Then, we have

$$
\xi_{\sigma} \stackrel{\sigma \searrow \zeta}{\longrightarrow} \frac{3^{1 / 3}}{2^{1 / 3} \eta^{1 / 3} \kappa_{2}^{1 / 3}}
$$

and

$$
(\sigma-\zeta)^{1 / 2} \overline{\tilde{w}}_{\sigma}(\bar{x}) \stackrel{\sigma \searrow \zeta}{\longrightarrow} \frac{3^{2 / 3} \eta^{1 / 3} \theta \kappa_{2}^{1 / 3}}{2^{5 / 3} \kappa_{0}} \bar{\chi}(\bar{x})\left(1-\bar{x}^{2}\right)
$$

as well as

$$
\lim _{\sigma \searrow \zeta} u_{\sigma}(x)=\theta, \quad \lim _{\sigma \searrow \zeta} \tilde{f}\left(u_{\sigma}(x)\right)=0
$$

in the sense of pointwise convergence.

The different limit behaviors for $\zeta=0$ and $\zeta>0$ can also be understood heuristically as follows. For $\zeta=\sigma=0$, there exists a plethora of nonlinear eigenfunction in $\mathcal{U}$, namely any function $u \in \mathcal{U}$ which satisfies $f(u) \equiv 0$ due to $\|u\|_{\infty}=u(0) \leq \theta$. In the case of $\zeta>0$, however, $\sigma=\zeta$ combined with $0 \leq u \leq \theta$ reduces (1) to $u=a * u$, but the only fixed points of the convolution operator are the constant functions. 


\section{Large Eigenvalues}

We start again with $\zeta=0$ but it turns out that our formal asymptotic results cover the case $\zeta>0$ as well. Since $\xi_{\sigma}$ is large for $\sigma \lesssim \eta$, we restate (39) as

$$
\left(\overline{\mathcal{A}}_{\xi} \bar{w}\right)(\bar{x})=\int_{\xi(\bar{x}-1)}^{\xi(\bar{x}+1)} a(y) \bar{w}\left(\bar{x}-\xi^{-1} y\right) \mathrm{d} y
$$

and employ the formal asymptotic expansion

$$
\left(\overline{\mathcal{A}}_{\xi} \bar{w}\right)(\bar{x}) \approx \bar{w}(\bar{x})+m \xi^{-2} \bar{w}^{\prime \prime}(x) .
$$

This formula involves

$$
m:=\frac{1}{2} \int_{-\infty}^{\infty} y^{2} a(y) \mathrm{d} y
$$

and holds for any fixed $-1<\bar{x}<+1$ thanks to Assumption 1. In combination with (38), we thus obtain the approximate ODE

$$
\theta+\lambda_{\sigma} \bar{w}_{\sigma}(\bar{x}) \approx \bar{w}_{\sigma}(\bar{x})+\xi_{\sigma}^{-2} m \bar{w}^{\prime \prime}(\bar{x}) \text { for } \bar{x} \in \bar{I}
$$

which admits the consistent solution

$$
\lambda_{\sigma} \approx 1-\xi_{\sigma}^{-2} \pi^{2} m, \quad \bar{w}_{\sigma}(\bar{x}) \approx d_{\sigma}(1+\cos (\pi \bar{x})), \quad d_{\sigma}=\frac{\theta \xi_{\sigma}^{2}}{\pi^{2} m} .
$$

These relations imply

$$
w_{\sigma}(x) \approx \frac{\theta \xi_{\sigma}^{2}}{\pi^{2} m} \chi \xi_{\sigma}(x)\left(1+\cos \left(\pi \xi_{\sigma}^{-1} x\right)\right)
$$

and give rise to the following claim.

Conjecture 3 (Asymptotics for $\zeta=0$ and $\sigma \approx \eta$ ) For any kernel a that decays sufficiently fast at infinity, we have

$$
(\eta-\sigma)^{1 / 2} \xi_{\sigma} \stackrel{\sigma \nearrow \eta}{\longrightarrow} \pi m^{1 / 2} \eta^{1 / 2}
$$

and

$$
(\eta-\sigma) \bar{w}_{\sigma}(\bar{x}) \stackrel{\sigma \nearrow \eta}{\longrightarrow} \eta \theta \bar{\chi}(\bar{x})(1+\cos (\pi \bar{x}))
$$

as well as $\left\|u_{\sigma}\right\|_{p} \sim(\eta-\sigma)^{-1-1 /(2 p)}$ for any $p \in[1, \infty]$. 
In the case of $\zeta>0$, the limit $\sigma \nearrow \zeta+\eta$ involves again the transformed kernel from Proposition 31, which satisfy $\tilde{a}_{\sigma} \rightarrow \tilde{a}_{\eta}$. We therefore expect that (47) and (48) remain true provided that $\sigma-\eta$ is replaced by $\tilde{\sigma}-\eta=\sigma-\zeta-\eta$ and $m$ is computed with $\tilde{a}_{\eta}$ instead of $a$.

We further emphasize that the scaling relations (47) and (48) are consistent with our numerical simulations in Figs. 2 and 3. The analytical justification of Conjecture 3 and the underlying approximation (45) is much harder than the rigorous derivation of (41) and (42) because the Taylor expansion is now applied to $\bar{w}_{\sigma}$ and requires uniform estimates of $\left\|\bar{w}_{\sigma}^{\prime \prime}\right\|_{p}$ for at least one $p \in[1, \infty]$. A similar problem concerns the linear eigenvalue problem from Sect. 2. In order to ensure the consistent approximation

$$
v_{\xi}(x) \approx-(\xi)^{-1 / 2} \chi \xi(x) \sin \left(\xi^{-1} \pi x\right)
$$

for the normalized eigenfunction from Proposition 1, we have to guarantee that $\left\|v_{\xi}^{\prime \prime}\right\|_{p} \leq C_{p} \xi^{\frac{1}{p}-\frac{5}{2}}$ holds for all large $\xi$ and some constant $C_{p}$ independent of $\xi$. We are, however, not aware of a corresponding reference. Moreover, the limit $\xi \rightarrow \infty$ is rather intricate due to a huge number of nearby eigenvalues that discretize the continuous spectrum of the limit operator $\mathcal{A}_{\infty} w=a * w$.

\section{References}

Anderson, T., Faye, G., Scheel, A., Stauffer, D.: Pinning and unpinning in nonlocal systems. J. Dyn. Differ. Equ. 28(3-4), 897-923 (2016). https://doi.org/10.1007/s10884-016-9518-6

Arapostathis, A.: A counterexample to a nonlinear version of the Krěn-Rutman theorem by R. Mahadevan. Nonlinear Anal. 171, 170-176 (2018)

Bakker, B., Scheel, A.: Spatial Hamiltonian identities for nonlocally coupled systems. Forum Math. Sigma 6, e22, 55 (2018). https://doi.org/10.1017/fms.2018.22

Burger, M., di Francesco, M., Franek, M.: Stationary states of quadratic diffusion equations with long-range attraction. Commun. Math. Sci. 11(3), 709-738 (2013). https://doi.org/10.4310/CMS.2013.v11.n3.a3

Burger, M., Haškovec, J., Wolfram, M.T.: Individual based and mean-field modeling of direct aggregation. Physica D 260, 145-158 (2013). https://doi.org/10.1016/j.physd.2012.11.003

Burger, M., Fetecau, R., Huang, Y.: Stationary states and asymptotic behavior of aggregation models with nonlinear local repulsion. SIAM J. Appl. Dyn. Syst. 13(1), 397-424 (2014). https://doi.org/10.1137/ 130923786

Chen, F., Herrmann, M.: KdV-like solitary waves in two-dimensional FPU-lattices. Discrete Contin. Dyn. Syst. Ser. A 38(5), 2305-2332 (2018). https://doi.org/10.3934/dcds.2018095

Crandall, M.G., Rabinowitz, P.H.: Bifurcation from simple eigenvalues. J. Funct. Anal. 8, 321-340 (1971). https://doi.org/10.1016/0022-1236(71)90015-2

Dautray, R., Lions, P.L.: Mathematical Analysis and Numerical Methods for Science and Technology. Volume 3 on Spectral Theory and Applications. Springer, Berlin (1990)

Dreyer, W., Herrmann, M.: Numerical experiments on the modulation theory for the nonlinear atomic chain. Physica D 237(2), 255-282 (2008)

Dreyer, W., Herrmann, M., Mielke, A.: Micro-macro transition for the atomic chain via Whitham's modulation equation. Nonlinearity 19(2), 471-500 (2006)

Faye, G., Scheel, A.: Existence of pulses in excitable media with nonlocal coupling. Adv. Math. 270, 400-456 (2015). https://doi.org/10.1016/j.aim.2014.11.005

Filip, A.M., Venakides, S.: Existence and modulation of traveling waves in particle chains. Commun. Pure Appl. Math. 51(6), 693-735 (1999)

Friesecke, G., Pego, R.L.: Solitary waves on FPU lattices. I. Qualitative properties, renormalization and continuum limit. Nonlinearity 12(6), 1601-1627 (1999). https://doi.org/10.1088/0951-7715/12/6/311 
Friesecke, G., Wattis, J.A.D.: Existence theorem for solitary waves on lattices. Commun. Math. Phys. 161(2), 391-418 (1994)

Haškovec, J., Oelz, D.: A free boundary problem for aggregation by short range sensing and differentiated diffusion. Discrete Contin. Dyn. Syst. Ser. B 20(5), 1461-1480 (2015). https://doi.org/10.3934/dcdsb. 2015.20.1461

Herrmann, M.: Unimodal wavetrains and solitons in convex Fermi-Pasta-Ulam chains. Proc. R. Soc. Edinb. Sect. A 140(4), 753-785 (2010). https://doi.org/10.1017/S0308210509000146

Herrmann, M.: Homoclinic standing waves in focusing DNLS equations. Discrete Contin. Dyn. Syst. Ser. A (DCDS-A) 31(3), 737-752 (2011). https://doi.org/10.3934/dcds.2011.31.737

Herrmann, M.: Oscillatory waves in discrete scalar conservation laws. Math. Models Methods Appl. Sci. (2012). https://doi.org/10.1142/S021820251200585X

Herrmann, M., Matthies, K.: Solitary waves in atomic chains and peridynamical media. Math. Eng. 1, 281-308 (2019). https://doi.org/10.3934/mine.2019.2.281

Herrmann, M., Matthies, K.: Stability of high-energy solitary waves in Fermi-Pasta-Ulam-Tsingou chains. Trans. Am. Math. Soc. 372(5), 3425-3486 (2019). https://doi.org/10.1090/tran/7790

Herrmann, M., Matthies, K.: Nonlinear and nonlocal eigenvalue problems: variational existence, decay properties, approximation, and universal scaling limits. Nonlinearity 33(8), 4046-4074 (2020). https:// doi.org/10.1088/1361-6544/ab8350

Herrmann, M., Mikikits-Leitner, A.: KdV waves in atomic chains with nonlocal interactions. Discrete Contin. Dyn. Syst. 36(4), 2047-2067 (2016). https://doi.org/10.3934/dcds.2016.36.2047

Iooss, G., James, G.: Localized waves in nonlinear oscillator chains. Chaos 15, 015113 (2005)

James, G.: Periodic travelling waves and compactons in granular chains. J. Nonlinear Sci. 22(5), 813-848 (2012). https://doi.org/10.1007/s00332-012-9128-3

Kaib, G.: Stationary states of an aggregation equation with degenerate diffusion and bounded attractive potential. SIAM J. Math. Anal. 49(1), 272-296 (2017). https://doi.org/10.1137/16M1072450

Kevrekidis, P.G.: The Discrete Nonlinear Schrödinger Equation, Springer Tracts in Modern Physics, vol. 232. Springer, Berlin (2009). https://doi.org/10.1007/978-3-540-89199-4

MacKay, R.S., Aubry, S.: Proof of existence of breathers for time-reversible or Hamiltonian networks of weakly coupled oscillators. Nonlinearity 7(6), 1623-1643 (1994)

Mahadevan, R.: A note on a non-linear Krein-Rutman theorem. Nonlinear Anal. 67(11), 3084-3090 (2007). https://doi.org/10.1016/j.na.2006.09.062

Omel'chenko, O.E.: The mathematics behind chimera states. Nonlinearity 31(5), R121-R164 (2018). https://doi.org/10.1088/1361-6544/aaaa07

Omel'chenko, O.E., Maistrenko, Y.L., Tassr, P.A.: Chimera states: the natural link between coherence and incoherence. Phys. Rev. Lett. 100, 044105 (2008). https://doi.org/10.1103/PhysRevLett.100.044105

Pankov, A.: Traveling Waves and Periodic Oscillations in Fermi-Pasta-Ulam Lattices. Imperial College Press, London (2005)

Pego, R., Van, T.: Existence of solitary waves in one dimensional peridynamics. J. Elast. 136(2), 207-236 (2019). https://doi.org/10.1007/s10659-018-9701-6

Rabinowitz, P.H.: Some global results for nonlinear eigenvalue problems. J. Funct. Anal. 7, 487-513 (1971). https://doi.org/10.1016/0022-1236(71)90030-9

Scheel, A., Tao, T.: Bifurcation to coherent structures in nonlocally coupled systems. J. Dyn. Differ. Equ. 31(3), 1107-1127 (2019). https://doi.org/10.1007/s10884-017-9613-3

Truskinovsky, L., Vainchtein, A.: Solitary waves in a nonintegrable Fermi-Pasta-Ulam chain. Phys. Rev. E 90, 042903:1-042903:8 (2014). https://doi.org/10.1103/PhysRevE.90.042903

Weinstein, M.I.: Excitation thresholds for nonlinear localized modes on lattices. Nonlinearity 12(3), 673691 (1999). https://doi.org/10.1088/0951-7715/12/3/314

Yasuda, H., Chong, C., Yang, J., Kevrekidis, P.G.: Emergence of dispersive shocks and rarefaction waves in power-law contact models. Phys. Rev. E 95, 062216 (2017). https://doi.org/10.1103/PhysRevE.95. 062216:1-5

Publisher's Note Springer Nature remains neutral with regard to jurisdictional claims in published maps and institutional affiliations. 